\title{
Biofilm Measurement, Site Specific Variation in Biofilm Production, Biofilm Typing: An Acinetobacter Trilogy
}

\author{
Baishali Chakraborty, Dibyendu Banerjee, and Shubhankar Mukherjee
}

\begin{abstract}
Acinetobacter baumannii is a member of the ESKAPE pathogens, notorious for causing multidrug resistant nosocomial infections worldwide. Biofilm has an important role in its persistence and spread in hospital environment, as well as its multidrug resistance potential. The present study was aimed at finding out whether the strength of Biofilm formation varies with infection of different organs / system. A total of 136 isolates of Acinetobacter baumannii were taken from a variety of samples. Strength of Biofilm formation, determined by Tissue culture plate method, was graded according to $O D$ value in ELISA reader as nil, moderate and strong. It was seen that association between variations in biofilm forming capacity depending on different sites of infection was statistically significant. We suggest biofilm typing by this method for this pathogen, as a potentially feasible alternative in countries with cost restrained healthcare set ups. Moreover, biofilm typing report would be more meaningful to the clinicians as well.

Index Terms-Acinetobacter; biofilm measurement; biofilm typing.
\end{abstract}

\section{INTRODUCTION}

In the bygone days, there was a group infamous for its criminal activity, especially murders, dreaded by all. The group was named by its own members as 'Ku-klux-clan'. They were so named because it phonetically resembled the sound of pressing the trigger of a gun. The modern day version or bacterial counterpart of the same clan are known as the 'ESKAPE' pathogens. They are responsible for most of the life threatening multidrug resistant (MDR) infections worldwide. The ESKAPE pathogens comprise of Enterococcus faecium, Staphylococcus aureus, Klebsiella pneumoniae, Acinetobacter baumannii, Pseudomonas aeruginosa, Enterobacter spp. [1]

If we look at the global patterns of emerging threats, as data obtained from CDDEP (Center for Disease Dynamics, Economics and Policy) for 2011-2014, the scenario is worrisome.

The percentage of Staphylococcus aureus that are methicillin resistant (MRSA) ranges from 40-59 in India, 6079 in Russian countries and 80-100 in some countries like Nigeria.

Percentage of extended spectrum beta lactamase (ESBL) producing Escherichia coli ranges from 80-100 in our country and 60-79 in China.

Carbapenem resistant Klebsiella pneumoniae ranges from $50-61 \%$ in India and $20-49.9 \%$ in China, USA, and Canada.

Published on September 6, 2019

Baishali Chakraborty is Associate professor, in the Department of Microbiology, Dr B C Roy Postgraduate Institute of Pediatric Sciences. Kolkata. India. (e-mail: drbaishalichakraborty@gmail.com).
In the year 2006, New Delhi Metallo betalactamase (NDM) emerged in India and later the bacteria carrying this gene became known as 'Superbugs', which became a worldwide threat. In this category, Pseudomonas aeruginosa was the 1st known superbug. Presently, Acinetobacter baumannii is an important member of this club among others.

Colistin resistance (chromosomal mediated), was there in the earlier days, which became nil due to long disuse of this drug. Colistin again came into use in recent years in cases where the bacteria were resistant to all other antibiotics.

The year 2015 saw the emergence of 1 st plasmid mediated polymyxin resistance mechanism, MCR-1, in Enterobacteriaceae. This gene, mcr-1, originated in China. [2] Then mor- 2 emerged in Brussels, followed by mor-3 in china again.

A large study was done to find the association between multi drug resistant (MDR) and extensively drug resistant (XDR) bacterial infections and in-hospital mortality rates among over 4000 patients hospitalized across India in 2015. These was one of the largest studies so far to measure the burden of antibiotics resistance in a low or middle income country. [3]

The study showed that the patients who acquired MDR bacterial infection were 1.5 times more likely to die, while patient who acquired XDR infections were 2.6 times more likely to die, compared to patients with similar susceptible infections. Among these, infections from gram negative bacteria showed higher mortality rates $(77.7 \%)$ than infections from gram positive bacteria which showed a lower mortality rates $(10.8 \%)$. The overall mortality rate was $13.1 \%$, with mortality as high as $29.0 \%$ among patients infected with Acinetobacter baumannii.

Acinetobacter baumannii was earlier considered to be only an opportunistic pathogen, causing ventilator associated pneumonia the patients admitted in the intensive care units. Unfortunately it has increased its virulence potential over the years, and is being increasingly the isolated from infections involving each and every organ. [4]

In 2017, 11th International symposium on the Biology of Acinetobacter was held in Sevilla, Spain (Acinetobacter 2017). The clear message which came out from the symposium was that carbapenem resistant strains of Acinetobacter baumannii are continuously spreading throughout the world. This spread is mainly by few clonal lineages -chief among them being ICL2 (International clonal lineage 2). Among the clonal lineages, three pan-European

Dibyendu Banerjee is Associate professor, in the Department of Microbiology, Calcutta National Medical College, Kolkata. India. (e-mail: drdibyendubanerjee2003@gmail.com).

Shubhankar Mukherjee is Assistant professor, Department of Community medicine, Calcutta National Medical College, Kolkata, India. (e-mail: shuvankarin@yahoo.com). 
lineages were already named as EU I, EU II, EU III in the 1990s. These carbapenem resistant Acinetobacter baumannii (CRAB) strains have also crossed the European territory and have reached USA, now being renamed by few as WW I, II,III - WW referring to worldwide.[5]

So one of the major problems for the medical community is to deal with health care associated infections by carbapenem resistant Acinetobacter baumannii. In this regard the works of Poirel and Nordmann are phenomenal. These pioneers stated that if one wants to tackle these pathogens, then the health care facilities are the best ones to start the fight. This is due to the fact that carbapenem resistant strains circulate mainly there- the community acquired isolates still somewhat lacking in MDR potential. [6]

It is well accepted that Acinetobacter baumannii shows the presence of dormant cell, a feature rarely noted in other gram negative bacteria. This accounts for it's environmental persistence - an ability to survive for a long time on abiotic surfaces under dry condition. This ability to colonize and to grow as biofilm has an important role in its persistence and spread in hospital environment. [7,8]

Biofilm is a microbially derived sessile community characterized by cells that are irreversibly attached to a substratum or interface or to each other, are embedded in a matrix of extracellular polymeric substances that they have produced, and exhibit an altered phenotype with respect to growth rate and gene transcription.[9]

There is a relationship between biofilm formation by a bacteria and consequences of disease produced by the pathogen. This may be due to several mechanisms such as

a) Detachment of cells or cell aggregates for medical device biofilm such as on catheter, resulting in blood stream and urinary tract infection.

b) Production of endotoxin.

c) Resistance to host immune system.

d) Resistance to antimicrobial drugs.

Thus in case of Acinetobacter baumannii its persistence and multidrug resistance potential is contributed to its biofilm forming ability. This study was done to have a deeper understanding of biofilm production in relation to different samples from which it is isolated.

\section{METHODS}

A total of 136 isolates of Acinetobacter baumannii (confirmed by Vitek 2) were taken from different clinical samples. The samples included sputum, endotracheal tube, pus, urine, blood (as given for culture in BactAlert), central venous catheter tips, and cerebrospinal fluid (CSF). Strength of biofilm formation by each isolate was measured by Tissue culture plate method. $[10,11,12,13]$ For this, two colonies of each isolate (from fresh subculture on MacConkey agar) were inoculated in Brain-heart infusion mixed with sucrose (BHIsuc). After bringing it to stationery phase, it was diluted 1 in $100 \mathrm{v} / \mathrm{v}$ in same broth and $200 \mathrm{ml}$ of it was poured in microtitre plates. The plates were incubated at $37^{0} \mathrm{c}$ for 24 hours. Each microtitre plate was washed with $0.2 \%$ phosphate buffered saline (PBS $0.2 \%$ ) to remove any planktonic bacteria. Then sodium acetate was used for fixation of biofilm, if formed. Then each plate was stained with crystal violet $(0.1 \% \mathrm{w} / \mathrm{v})$ for 30 minutes. Reading was taken in ELISA reader, keeping primary filter at $570 \mathrm{~nm}$ and secondary filter at 0 . Strength of biofilm formation was graded as per Christensen et al as follows: $[12,13]$

OD value $<0.120$ - No biofilm formation;

OD value $0.120-0.240$ - Moderate biofilm formation; OD value $>0.240$ - Strong biofilm formation.

\section{RESULTS}

(i) Maximum number of isolates infecting CVC tips were strong biofilm producers $(70 \%)$, followed closely by $\mathrm{E}$ $\mathrm{T}$ tube isolates $(69.5 \%)$.

(ii) The isolates from bloodstream, urine and sputum showed all the three patterns, namely, biofilm non-producer, moderate biofilm producer and strong biofilm producer.

(iii) Interestingly, from pus sample isolates no strong biofilm producer was found, and from CSF isolates no nonproducer of biofilm emerged.

TABLE I: ASSOCIATION BETWEEN BIOFILM STRENGTH AND INFECTION OF DIFFERENT SITES BY A. BAUMANNII. (THE FIGURES IN PARENTHESIS INDICATE PERCENTAGE)

\begin{tabular}{lllll}
\hline \hline & $\begin{array}{l}\text { No } \\
\text { biofilm }\end{array}$ & $\begin{array}{l}\text { Moderate } \\
\text { biofilm }\end{array}$ & Strong biofilm & Total \\
\hline Sputum & $4(28.5)$ & $4(28.5)$ & $6(42.8)$ & 14 \\
ET Tube & $12(26.0)$ & $2(4.3)$ & $32(69.5)$ & 46 \\
Pus & $4(50.0)$ & $4(50.0)$ & - & 8 \\
Urine & $2(12.5)$ & $10(62.5)$ & $4(25.0)$ & 16 \\
Blood & $4(4.2)$ & $14(50.0)$ & $10(25.7)$ & 28 \\
CVC & $2(10.0)$ & $4(20.0)$ & $14(70.0)$ & 20 \\
CSF & - & $2(50.0)$ & $2(50.0)$ & 4 \\
Total & 28 & 36 & 68 & 136 \\
\hline \hline
\end{tabular}

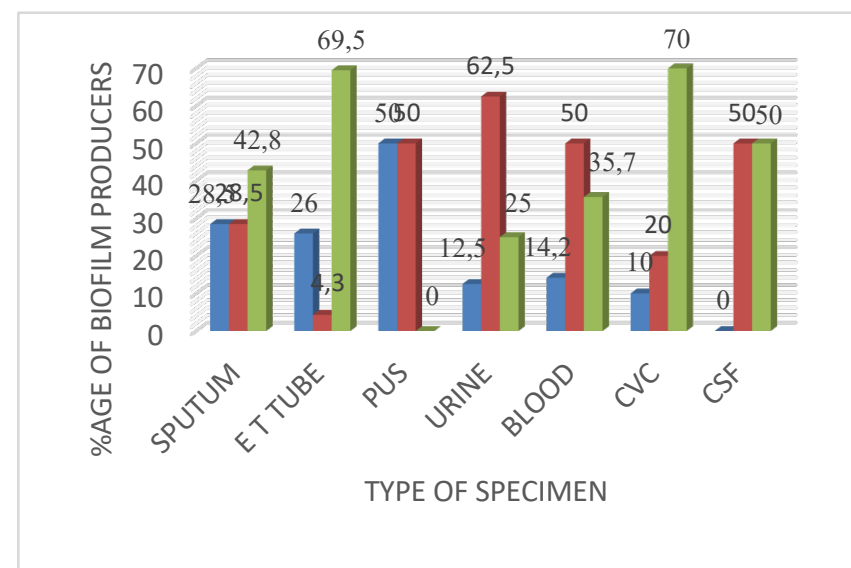

NO BIOFILM — MODERATE BIOFILM — STRONG BIOFILM

Fig. 1. Association between biofilm strength and infection of different sites by $A$. baumannii.

STATISTICAL ANALYSIS: Chi-square test was applied for comparing categorical data at 5\% significance level. It was seen that association between variation in biofilm forming 
capacity depending on different sites of infection was not random, but statistically significant

$[\chi 2(12)=41.99 . p=0.00003]$.

\section{DISCUSSION}

It is evident from the results that most of the isolates infecting CVC tips and endotracheal tubes are strong biofilm producers $(70 \%$ and $69.5 \%$ respectively). Urinary tract pathogens were mostly moderately strong biofilm producers. In bloodstream isolates all variants were present and we attribute this distribution (35.7\% strong/50.0\% moderate/ $14.2 \%$ nil) to any organotropism yet to be developed among circulating strains, although biofilm producers were clearly higher than non-producers.

Two samples which we want to categorically mention are pus and CSF. In the former, for the pyogenic strains strong biofilm formation was not mandatory. In the latter case, it seems that to invade CSF the strains have to produce biofilms.

Sputum sample isolates showed all three patterns of biofilm producing capacity, and similar to bloodstream isolates biofilm producers (strong plus moderate) were clearly in excess than the non-producers.

In the article titled "Resources for genetic and genomic analysis of Emerging pathogen Acinetobacter baumannii", the authors commented that $A$. baumannii infections has become the scourge of healthcare facilities worldwide, and eliminating such infections requires a deeper understanding of the factors which enable the pathogen to persist in hospital environments. [14] This study has tried to look into the cause of biofilm formation from a new angle. As there seems to be a strong association between Acinetobacter baumannii isolates from different sources of infection and strength of biofilm formation by the isolates, it may be implied that this bacteria forms strong biofilm, moderate biofilm, or none at all - depending on the organ they are going to infect. In other words, this study hints, albeit in a preliminary manner, at a link between organotropism of different strains of Acinetobacter baumannii and production of biofilm by them.

If one wants to control a dangerous pathogen like this, which is not only multidrug resistant but also a persistent pathogen causing nosocomial infection, epidemiological tracking is mandatory. The tools of molecular epidemiology are Pulsed Field Gel Electrophoresis (PFGE), Matrix assisted laser desorption / ionization time of flight mass spectrometry ( Maldi- ToF- MS), and others. That's exactly what the molecular epidemiologists do. They identify the different genetic strains present in different regions with their molecular tools. These tools, however, although razor sharp in precision and accuracy, are costly, and require a certain amount of technical expertise. Unfortunately enough, these technologies are unavailable in primary health care facilities and even in many tertiary health care facilities in low- and middle income countries like India where cost restriction and man power scarcity are problems which go hand in hand.

Accepting biofilm as an important virulence factor, and because of variation of pattern changing with different sites of infection as shown by us, we suggest Biofilm typing for this organism. The method is easy, effective in a costrestricted scenario, and can be done with simple instrument like an ELISA reader available in every laboratory set up, thanks to Dengue and other arboviral diseases. These diseases are rampant in the countries where cost restraints are maximum, and the government supplies ELISA readers to each health care facility free of cost for diagnosis of these arboviral threats.

In no way this biofilm typing is to replace the modern molecular methods of typing like Maldi-TOF-MS, PFGE and others. Molecular epidemiologists with their razor-sharp technologies should obviously be there, as an ultimate confirmatory backdrop whenever and wherever available. We are just suggesting a cheap, globally available easy alternative of typing method, available and affordable in low- and middle income countries upto the primary healthcare level.

Finally, all these endeavours are to control a deadly pathogen and this needs a teamwork. In this team if the clinician is not involved, then the whole exercise is bound to be futile. They are the ones who face the infection in a patient on first hand basis. Molecular typing of strains and terminology may bear little significance to them, in their busy schedule. For example, instead of trying to alert them by telling "Escherichia coli O157:H7" is present in your hospital, a far more useful method would be telling them that a dangerous strain of Escherichia coli causing hemorrhagic colitis and Hemolytic uraemic syndrome is present in this hospital.

Similarly, biofilm typing can be helpful here as well. Biofilm is a term which is not only a common knowledge to medical microbiologists, but is very well recognized by the clinicians as well. Thus, biofilm typing is written in a language which the clinicians interpret with ease. Hence, their involvement would make the team - a so called team of "Mission Impossible" - strong enough to control the spread of this emerging pathogen on the face of the globe more securely.

FUNDING: None.

CONFLICTS OF INTEREST: None declared.

\section{REFERENCES}

[1] Tommasi R, Brown DG, Walkup GK, Manchester JI, Miller AA. ESKAPEing the labyrinth of antibacterial discovery. Nat Rev Drug Discov. 2015;14:529-542.

[2] Liu YY, Wang Y, Walsh TR, Yi LX, Zhang R, Spencer J et al. Emergence of plasmid-mediated colistin resistance mechanism MCR1 in animals and human beings in China: a microbiological and molecular biological study. Lancet Infect Dis. 2016 Feb;16(2):161-68. doi: 10.1016/S1473-3099(15)00424-7.

[3] Gandra S, Tseng KK, Arora A, Bhowmik B, Robinson ML, Panigrahi B, Laxminarayan R, Klein EY. The Mortality Burden of Multidrugresistant Pathogens in India: A Retrospective, Observational Study. Clin Infect Dis. 2015;69(4):563-70.

[4] Chakraborty B, Banerjee D, Chakraborty B. Acinetobacter baumannii: No more a choosy intruder? Indian J Med Sci. 2011;65:344-48.

[5] Adams-Haduch JM, Onuoha EO, Bogdanovich T, Tian GB, Marschall J, Urban CM, Spellberg BJ, Rhee D, Halstead DC, Pasculle AW, Doi Y. Molecular epidemiology of carbapenem-nonsusceptible Acinetobacter baumannii in the United States. J Clin Microbiol. 2011;49(11):3849-54

[6] Poirel L, Nordmann P. Carbapenem resistance in Acinetobacter baumannii: mechanisms and epidemiology. Clin Microbiol Infect. 2006;12:826-836.

[7] Longo F, Vuotto C, Donelli G. Biofilm formation in Acinetobacter baumannii. New Microbiol. 2014;37:119-127.

[8] Rao RS, Karthika RU, Singh SP, Shashikala P, Kanungo R, Jayachandran $\mathrm{S}$ et al. Correlation between biofilm production and multiple drug resistance in imipenem resistant clinical isolates of Acinetobacter baumannii. Indian J Med Microbiol. 2008;26: 333-337. 
[9] Donlan R. Costerton J. Biofilms: Survival Mechanisms of Clinically Relevant Microorganisms. Clin Microbiol Rev. 2002;15:167-193.

[10] Mathur T, Singhal S, Khan S, Upadhyay DJ, Fatma T, Rattan A. Detection of biofilm formation among the clinical isolates of staphylococci: an evaluation of three different screening methods. Indian J Med Microbiol. 2008;24:25-29.

[11] Kwasny S, Opperman J. Static biofilm cultures of Gram-positive pathogens grown in a microtiter format used for anti-biofilm drug discovery. Curr Protoc Pharmacol. 2010 Sep 1. https://www.ncbi.nlm.nih.gov/pmc/articles/PMC3272335.

[12] Christensen GD, Simpson WA, Bisno AL, Beachey EH. Adherence of slime-producing strains of Staphylococcus epidermidis to smooth surfaces. Infect Immun. 1982;37:318-26.

[13] Panda P, Chaudhary U, Dube S. Comparison of four different methods for detection of biofilm formation by uropathogens. Indian J Pathol Microbiol. 2016;59:177-179.

[14] Gallagher LA, Ramage E, Weiss EJ, Radey M, Hayden HS et al. Resources for Genetic and Genomic Analysis of Emerging Pathogen Acinetobacter baumannii. J Bacteriol. 2015;197(12):2027-35. 